\title{
SZ-685C exhibits potent anticancer activity in both radiosensitive and radioresistant NPC cells through the miR-205-PTEN-Akt pathway
}

\author{
DUJUAN WANG, SUMEI WANG, QIN LIU, MENGYAO WANG, CHUNHUA WANG and HUILING YANG \\ Department of Pathophysiology, Zhongshan School of Medicine, Sun Yat-Sen University, \\ Guangzhou, Guangdong 510080, P.R. China
}

Received December 14, 2012; Accepted January 17, 2013

DOI: $10.3892 /$ or.2013.2376

\begin{abstract}
Radioresistance is a major obstacle to the treatment of human nasopharyngeal carcinoma (NPC). Emerging evidence has demonstrated that miRNAs are involved in cancer therapy resistance. Our research group established the radioresistant NPC cell line CNE2R derived from the CNE2 cell line, and demonstrated that irradiation-induced miR-205 determined the resistance of NPC through directly targeting PTEN. However, specific inhibitors targeting miRNAs are largely undetermined. SZ-685C was expected to abrogate the radioresistance of CNE2 cells through the miR-205-PTENAkt pathway. SZ-685C exhibited a similar cytotoxic effect on both cell lines, and we demonstrated that both intrinsic and extrinsic pathways were activated by SZ-685C in the cell lines. Importantly, the miR-205-PTEN-Akt pathway was the key cell signaling pathway activated in the CNE2R cells upon SZ-685C treatment; however, the Stat3-Jab1-p27 pathway might participate in the pro-apoptotic effect in CNE2 cells but not in CNE2R cells. SZ-685C is a promising anticancer agent for treatment of NPC, and it exhibited pro-apoptotic activity in both radiosensitive and radioresistant NPC cells. Although the mechanisms between the two cell lines were not identical, the pro-apoptotic effects were similar between the two cell lines.
\end{abstract}

\section{Introduction}

Human nasopharyngeal carcinoma (NPC) is one of the most common cancers in Southern China (1). The highest incidence is found among Southern Chinese ( 25-30 per 100,000 individuals per year), in particular those of Cantonese origin (2). Although NPC is a relatively radiosensitive disease, the thera-

Correspondence to: Professor Huiling Yang, Department of Pathophysiology, Zhongshan School of Medicine, Sun Yat-Sen University, Guangzhou, Guangdong 510080, P.R. China

E-mail: hlyangsums@hotmail.com

Key words: human nasopharyngeal carcinoma, radioresistance, apoptosis, miR-205, Akt, Stat3 peutic effect remains unsatisfactory due to radioresistance (3). Aiming to identify the mechanisms of radioresistance, our research group established a radioresistant NPC cell line (CNE2R) derived from the CNE2 cell line. In recent years, epigenetic changes in miRNAs have attracted increasing attention as they regulate a wide variety of drug-resistant genes (4). Emerging evidence suggests that miRNAs are involved in cancer therapy resistance $(5,6)$, and several studies have focused on alterations in proteins and genes which are regulated by miRNAs in order to explain the mechanisms of resistance $(7,8)$. We demonstrated that irradiation-induced miR-205 determined the resistance of NPC by targeting PTEN (9). However, specific inhibitors targeting miRNAs remain largely undetermined.

SZ-685C, an anthracycline analogue isolated from the secondary metabolites of the mangrove endophytic fungus no. 1403 collected from the South China Sea (10), was found to exhibit an anticancer effect on breast cancer in vivo and in vitro involving the inhibition of Akt/FOXO (11). The upregulations of Akt and FOXO are linked to drug resistance (12). These studies suggest that SZ-685C is a potential anticancer drug candidate for cancer therapy resistance. To date, no studies have directly investigated whether NPC cells are sensitive to SZ-685C, particularly the radioresistant nasopharyngeal carcinoma cell line CNE2R. In the present study, we confirmed the anticancer activity of SZ-685C in both CNE2 and CNE2R cells. Furthermore, we demonstrated, for the first time, that protein changes in anticancer pathways and also potent miRNA changes participated in the anticancer effect of SZ-685C on the two NPC cell lines.

\section{Materials and methods}

Preparation of SZ-685C. SZ-685C was obtained from the Guangdong Province Key Laboratory of Functional Molecules in Oceanic Microorganisms (Sun Yat-sen University). This product was dissolved in dimethylsulphoxide (DMSO) at a concentration of $50 \mathrm{nM}$ as a stock solution and diluted according to experimental requirements when used.

Cell culture. Human nasopharyngeal carcinoma cell line CNE2 was obtained from the Cancer Center, Sun Yat-sen 
University (Guangzhou, China), and the stable radioresistant NPC cell line CNE2R was established in our laboratory. The two cell lines were cultured in RPMI-1640 medium containing $10 \%$ fetal bovine serum with penicillin-streptomycin sulfate. All cell lines were incubated at $37^{\circ} \mathrm{C}$ in an atmosphere of $5 \% \mathrm{CO}_{2}$.

Cell viability and colonyformation assays.MTT (3,4,5-dimethylthiazol-2-yl)-2-5-diphenyltetrazolium bromide) assay was used to determine the effect of SZ-685C on cell viability. The cells were seeded in $200 \mu \mathrm{l}$ media for $12 \mathrm{~h}$ and incubated with $0.1,0.5,1,5,10,20,30,40$ and $50 \mu \mathrm{M} \mathrm{SZ}-685 \mathrm{C}$ for 24,48 and $72 \mathrm{~h}$. Cells were then incubated with MTT (Sigma-Aldrich, St. Louis, MO, USA) reagent for $4 \mathrm{~h}$, lysed with DMSO and quantitated using a plate reader at $570 \mathrm{~nm}$.

Clonogenic assays were performed by seeding cells into 6-well plates, allowing adherence overnight, and the culture medium was replaced with $3 \mathrm{ml}$ of fresh media containing SZ-685C at specified concentrations. After incubation for 14 days, the cells were fixed with methanol, they were then stained with $0.2 \%$ Giemsa (Sigma-Aldrich), and finally colonies consisting of $>50$ cells were counted.

Apoptotic morphologic changes. CNE2 and CNE2R cells were plated into 6 -well plates at a density of $2 \times 10^{5}$ cells/well overnight, and then SZ-685C was added at final concentrations of $0,10,20$ and $30 \mu \mathrm{M}$. After $48 \mathrm{~h}$, cells were stained with Hoechst 33342 (Molecular Probes, Life Technologies, USA) at $10 \mu \mathrm{g} / \mathrm{ml}$ for $15 \mathrm{~min}$, fixed with methanol and subsequently washed with PBS. A phase-contrast fluorescence microscope was used to observe apoptotic morphologic changes.

Determination of cell cycle and apoptosis. Cells $\left(2 \times 10^{5}\right.$ well) in 6-well plates were maintained with 20 and $30 \mu \mathrm{M}$ of $\mathrm{SZ}-685 \mathrm{C}$ for $48 \mathrm{~h}$. The cells were harvested by centrifugation and fixed in cold $70 \%$ ethanol at $4^{\circ} \mathrm{C}$ overnight ( $\geq 12 \mathrm{~h}$ ). Fixed cells were washed with PBS and stained with propidium iodide containing RNase A at $10 \mu \mathrm{g} / \mathrm{ml}$. Cells were collected by flow cytometry (FACSCalibur, Becton-Dickinson, San Jose, CA, USA) and analyzed using ModFit Software (Verity Software House Inc., Topsham, ME, USA).

For apoptosis experiments, cells were seeded and cultured as above. Cell apoptosis was determined by flow cytometry. Cells were stained with Annexin-V-FITC and propidium iodide (PI), using an assay kit from Roche as described by the manufacturer (Roche Diagnostics, Indianapolis, IN, USA).

Western blotting. Cells were seeded into $60-\mathrm{mm}$ dishes overnight and then treated with SZ-685C at different concentrations for 12, 24 or $48 \mathrm{~h}$. Including the floating cells, all the cells were harvested and lysed in 1X sampling buffer (Cell Signaling Technology, Danvers, MA, USA) and protease inhibitor cocktail (Roche). The concentrations of these lysates were determined by a bicinchoninic acid protein assay kit (Pierce Biotech, Rockford, IL, USA). Equivalent amounts of proteins were loaded on a 10,12 or $15 \%$ polyacrylamide SDS gel and were electrophoresed for 1.5-2 $\mathrm{h}$. Proteins were then transferred from the gel to polyvinylidene fluoride (PVDF) membranes (EMD Millipore, Billerica, MA, USA). Membranes were blocked with fat-free milk powder (Roche)
$(5 \%, \mathrm{w} / \mathrm{v})$ and Tween $20(1 \%, \mathrm{w} / \mathrm{v})$ in TBS-T for $1 \mathrm{~h}$. Protein bands were detected by exposing the membranes to primary antibodies overnight at $4^{\circ} \mathrm{C}$. After washing and secondary antibody incubation, the signal from the target protein band was visualized on X-ray film using ECL western blotting reagents (Biyuntian, Beijing, China).

The following primary antibodies were used: a 1:1,000 dilution of rabbit PTEN (Santa Cruz Biotechnology, Inc., Santa Cruz, CA, USA), total Akt (Cell Signaling Technology), 14-3-3ל (Abcam Ltd., Hong Kong); a 1:1,000 dilution of mouse caspase-3, Stat3, p27 (Cell Signaling Technology), Bcl-2/Bax (BD Pharmingen, BD Biosciences, San Diego, CA, USA), caspase-4, Jab1 (Abcam); a 1:500 dilution of mouse monoclonal caspase-8, caspase-9 (Santa Cruz Biotechnology); a $1: 10,000$ dilution of mouse monoclonal $\beta$-actin (Sigma) and an HRP-conjugated secondary antibody (Cell Signaling Technology) followed by enhanced chemiluminescence detection.

$q R T-P C R$. Total RNA was extracted with the TRIzol reagent (Invitrogen, Carlsbad, CA, USA). For miRNA quantification, the cDNA was synthesized by SuperScript ${ }^{\circledR}$ III First-Strand (Invitrogen) and Platinum ${ }^{\circledR} \mathrm{SYBR}^{\circledR}$ Green qPCR SuperMix-UDG (Invitrogen). The special miR-205 and miR-24 were designed by RiboBio (Guangzhou, China). Expression levels were normalized to that of U6 rRNA and then converted into relative values calculated by the comparative CT method. The mRNA levels for PTEN and Akt were measured by a method similar to miRNA quantification. $\beta$-actin was used as an internal control for normalization. qRT-PCR reactions were performed with the following primers: PTEN forward, 5'-TGGATTCGACTTAGACTTGACCT-3'; reverse, 5'-TTTGGCGGTGTCATAATGTCTT-3'; Akt1 forward, 5'-TCTTTGCCGGTATCGTGT-3'; reverse, 5'-TGTC ATCTTGGTCAGGTGGT-3'. All quantitative real-time PCR was performed using the iQ5 Bio-Rad Real-Time PCR system (Bio-Rad Laboratories, Hercules, CA, USA).

Statistical analysis. At least three independent experiments were performed for statistical evaluation. Data are presented as means $\pm \mathrm{SD}$. The statistical significance was evaluated using the Student's t-test. A P-value of $<0.05$ was considered to indicate a statistically significant result.

\section{Results}

Cytotoxic effect of SZ-685C on CNE2 and CNE2R cells. The viabilities of CNE2 and CNE2R cells decreased in a dose- and time-dependent manner following treatment with SZ-685C (Fig. 1A). As shown in Fig. 1B, after treatment with SZ-685C for 24,48 and $72 \mathrm{~h}$, the calculated $\mathrm{IC}_{50}$ value was $46.89,14.13$ and $8.97 \mu \mathrm{M}$ for CNE2 cells and 69.11, 17.86 and $8.94 \mu \mathrm{M}$ for CNE2R cells, respectively. We further determined the clonogenic abilities of the two cell lines treated with SZ-685C (Fig. 1C) and found that there were no significant differences between CNE2 and CNE2R cells.

Effects of SZ-685C on apoptosis and the cell cycle. To confirm whether the decreased viability of CNE2 and CNE2R cells induced by SZ-685C was associated with apoptosis, we 
A

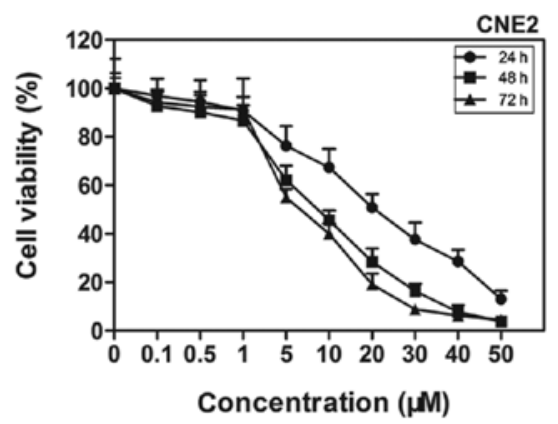

B

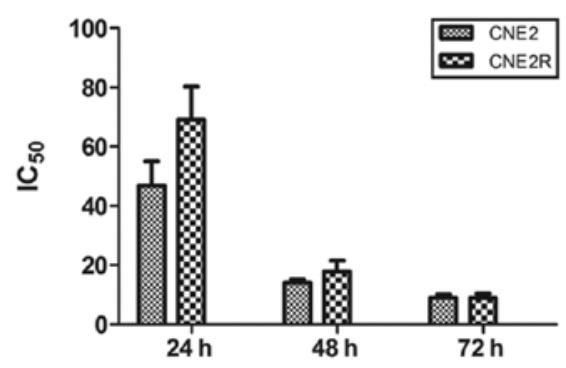

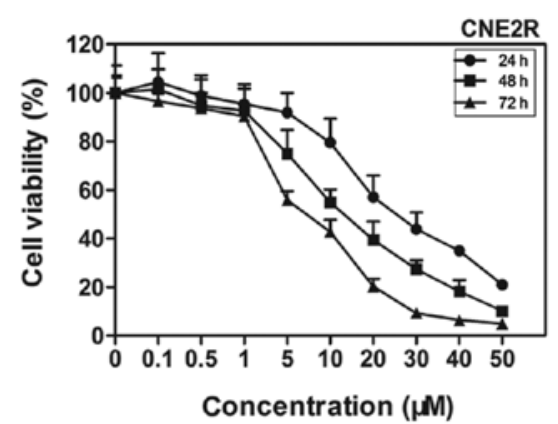

C

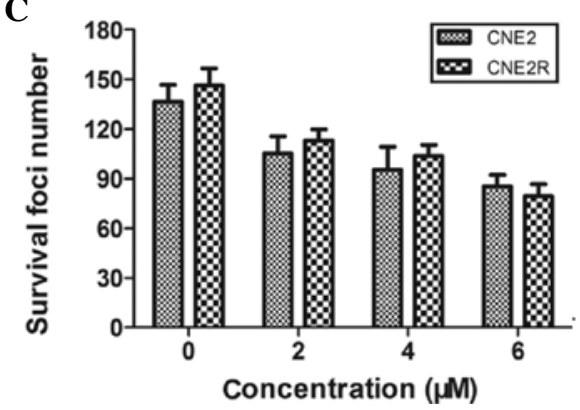

Figure 1. Effects of SZ-685C on the growth of NPC cell lines. (A) CNE2 and CNE2R cells were incubated with various concentrations of SZ-685C for 24, 48 and $72 \mathrm{~h}$, and cell viability was quantified by MTT assay. (B) $\mathrm{IC}_{50}$ values of SZ-685C for both cell lines are shown in the histogram. (C) Representative results of the colony formation assay of the CNE2 and CNE2R cells. All the data are presented as means \pm SD.

imaged the morphologic changes of the two cell lines treated with SZ-685C for $48 \mathrm{~h}$. We observed nuclear fragmentation and bright blue nuclei (Fig. 2A). In CNE2 cells, the percentage of apoptotic cells was $\sim 70 \%$, and the percentage in CNE2R cells was $72 \%$. The data indicated that the pro-apoptotic effect of SZ-685C on the two cell lines was not significantly different. We next analyzed the number of apoptotic cells by flow cytometry. In the CNE2 cells, treatment with 20 and $30 \mu \mathrm{M}$ SZ-685C for $48 \mathrm{~h}$ resulted in 29.18 and $75.83 \%$ of the cells undergoing apoptosis, respectively. For the CNE2R cells, the percentages of apoptotic cells were 32.65 and $48.97 \%$, respectively (Fig. 2B).

Cell cycle progression using flow cytometry was determined. SZ-685C induced cell cycle arrest at the G2/M phase in CNE2 cells, and the percentage of cells in the $\mathrm{G} 2 / \mathrm{M}$ phase increased from $5.5 \%$ at $0 \mu \mathrm{M} \mathrm{SZ}-685 \mathrm{C}$ to $14.93 \%$ at $20 \mu \mathrm{M}$ SZ-685C at $24 \mathrm{~h}$. At the same time, in the CNE2R cells, similar results were found showing that the proportion of cells in the $\mathrm{G} 2 / \mathrm{M}$ phase increased from 5.43 to $15.17 \%$ (Fig. 2C).

Activation of apoptotic pathways by SZ-685C. In order to elucidate the exact apoptotic pathways involved, we assessed the levels of several key caspases. As shown in Fig. 3, in CNE2 cells, the levels of procaspase- $3,-8$ and -4 decreased in an obvious dose-dependent manner, and the level of cleaved caspase- 4 increased in the same manner. We also detected changes in these proteins in the CNE2R cells, and found similar data except procaspase- 9 which was decreased in the CNE2R cells. The data indicated that SZ-685C induced apoptosis which involved the intrinsic and extrinsic pathways in both cell lines.

We determined the levels of Bcl-2 and Bax in both cell lines following SZ-685C treatment for $48 \mathrm{~h}$. The level of Bcl-2 obviously decreased while the level of Bax was increased in the CNE2 and CNE2R cell lines (Fig. 3).

SZ-685C induces apoptosis via the miR-205-PTEN-AKT pathway. To elucidate the anticancer mechanisms involved in the pro-apoptotic effect of SZ-685C on NPC cells which are mediated through the Akt pathway, the expression of PTEN and $\mathrm{Akt}$ and the phosphorylation status of Akt were assessed (Fig. 4B). SZ-685C treatment for $48 \mathrm{~h}$ resulted in a decrease in Akt and phosphor-Ser473-Akt and an increase in PTEN in the CNE2 and CNE2R cell lines. We found that after treatment with SZ-685C at the concentration of $40 \mu \mathrm{M}$ for $48 \mathrm{~h}$ phosphor-Ser473-Akt decreased in the CNE2 cells; however, in CNE2R cells it was altered at $10 \mu \mathrm{M}$. The mRNA levels of Akt1 in the two cell lines were not identical; the level was higher in the CNE2R cells when compared with that in the CNE2 cells. Following treatment with SZ-685C for $48 \mathrm{~h}$, the mRNA level of Akt1 was decreased more significantly in CNE2R cells than that in CNE2 cells (Fig. 4A).

We subsequently detected the mRNA expression of PTEN and miR-205 (Fig. 4A). As predicted, the tendency was similar, while the difference between the two cell lines was noted. The initial mRNA level of PTEN in the CNE2R cells without SZ-685C was much lower when compared with that in CNE2 cells, while the level of miR-205 was much higher in CNE2R cells than that in the CNE2 cells. After treatment with SZ-685C for $1 \mathrm{~h}$, the level of miR-205 decreased in the CNE2R cells, and the tendency was significant. However, in CNE2 cells, the decrease in the level of miR-205 was slight at $4 \mathrm{~h}$ following SZ-685C treatment. A similar trend was determined for PTEN. The increased level of PTEN in CNE2R cells was higher than that in CNE2 cells upon SZ-685C treatment for $4 \mathrm{~h}$. 
A
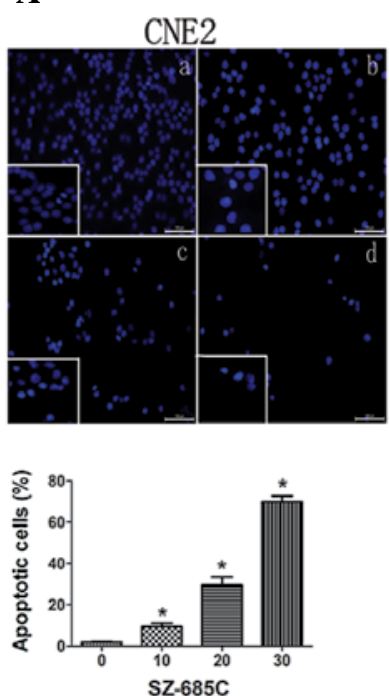

C
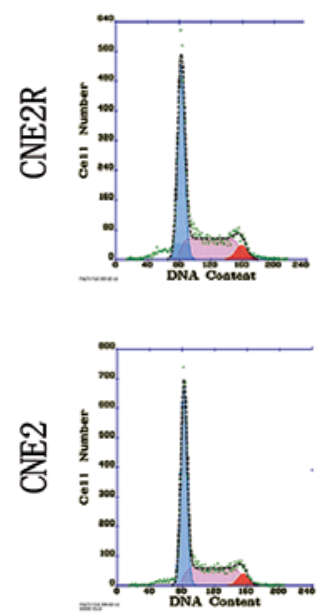
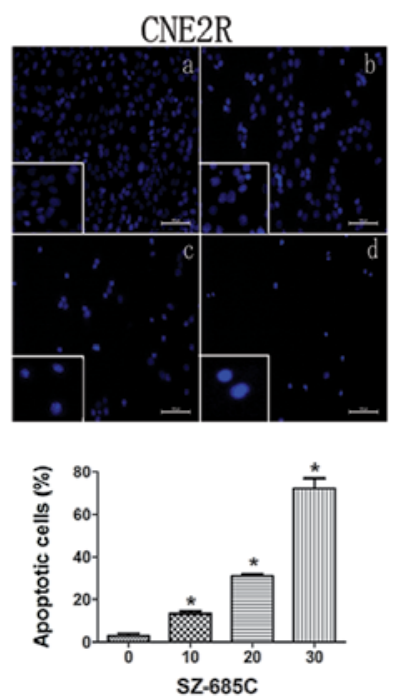

B
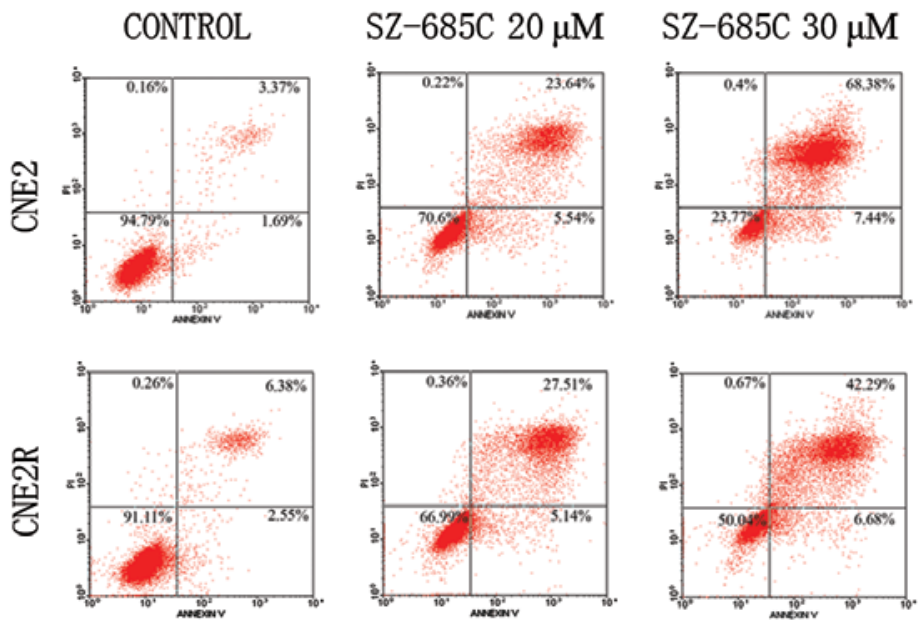
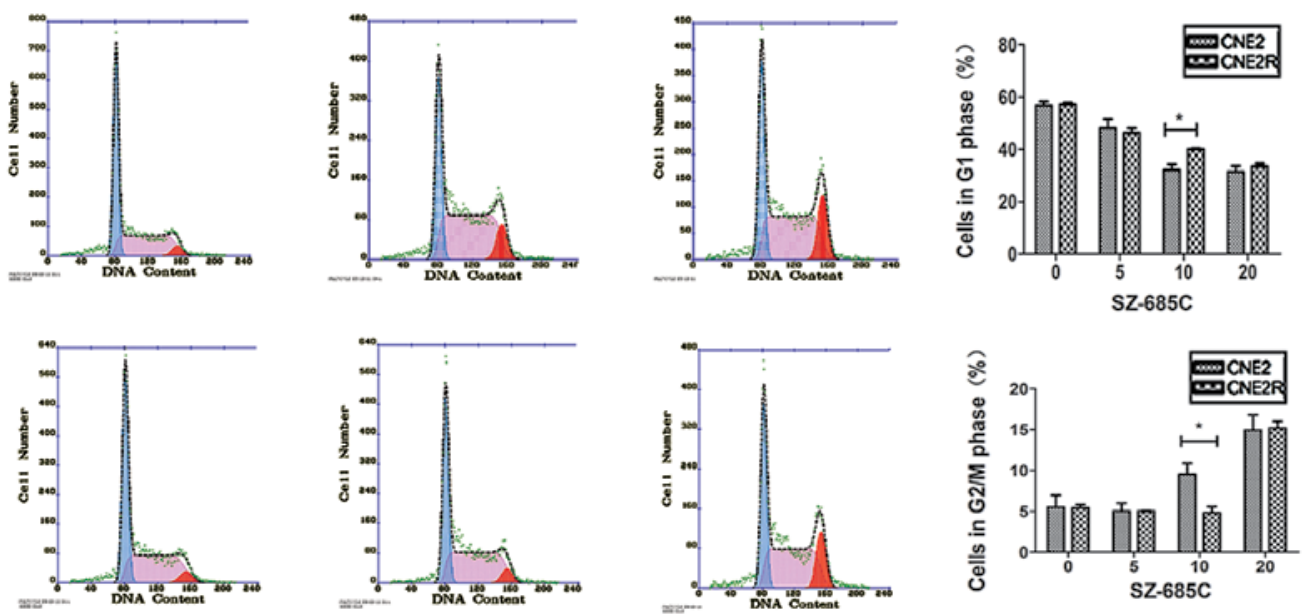

Figure 2. Effects of SZ-685C on apoptosis and cell cycle arrest. (A) CNE2 and CNE2R cells were stained with $10 \mu \mathrm{g} / \mathrm{ml}$ of Hoechst 33342 for $15 \mathrm{~min}$ at $48 \mathrm{~h}$ post-SZ-685C treatment at different concentrations (a; control; b, $10 \mu \mathrm{M} \mathrm{SZ-685C}$; c, $20 \mu \mathrm{M} \mathrm{SZ}-685 \mathrm{C}$, d, $30 \mu \mathrm{M}$ SZ-685C). Samples were visualized under a fluorescence microscope (x10). For quantification, Hoechst-positive nuclei were counted (at least 400 cells were counted for each condition. Bottom panel: quantification of apoptotic cells is shown. (B) Cells were exposed to SZ-685C for $48 \mathrm{~h}$. Cells were collected and subjected to Annexin V-FITC/PI staining and analyzed by flow cytometry. (C) CNE2 and CNE2R cells were treated with SZ-685C for $24 \mathrm{~h}$, then stained with PI and analyzed by flow cytometry. The percentages of CNE2 and CNE2R cells in the G1 and G2/M phase are shown. Data represent three independent experiments, means \pm SD; ${ }^{*} \mathrm{P}<0.05$.

SZ-685C induces changes in the Stat3-Jabl-p27 pathway in CNE2 cells but not in CNE2R cells. We screened the proteins involved in apoptosis and survival. We found different changes in $\mathrm{p} 27$, Stat 3 and $14-3-3 \zeta$ in the two NPC cell lines. We discovered that in CNE2 cells treated with SZ-685C for $12 \mathrm{~h}$, the level of Stat 3 decreased and the level of 14-3-3 $\zeta$ increased in a dose-dependent manner, and the level of p27 increased after treatment with SZ-685C for 48 h (Fig. 4D). In an opposing manner, the levels of the three proteins did not change in CNE2R cells under the same conditions.

$S Z-685 C$ regulates $m i R-24$ expression. After establishment of the radioresistant NPC cell line, CNE2R, we screened for different miRNAs between CNE2 and CNE2R. miR-205 was one of the 9 miRNAs with differential expression and it was the most significant one. Thus, we aimed to ascertain whether expression of other miRNAs could be regulated by SZ-685C. We found that miR-24 expression was obviously altered after treatment with SZ-685C for 12 and $24 \mathrm{~h}$, implying that miR-24 may participate in the pro-apoptotic effect of SZ-685C on both cell lines. We also found that the change was much more significant in CNE2 cells than that in CNE2R cells (Fig. 4C).

\section{Discussion}

Radiotherapy is a major treatment option for NPC; yet, radioresistance is the main obstacle to the treatment of such patients. Therefore, understanding the mechanisms of radioresistance and developing compounds to reverse this resistance are challenging for improving the prognosis of NPC patients. Based on the radioresistant cell line $\mathrm{CNE} 2 \mathrm{R}$, we previously reported that miR-205 targets tumor-suppressor PTEN and consequently activates the PI3K/Akt pathway leading to increase radioresistance of NPC, indicating that the miR-205-PTEN pathway may determine the threshold for overcoming radioresistance in NPC cells (9). It has been demonstrated that estrogen receptor 


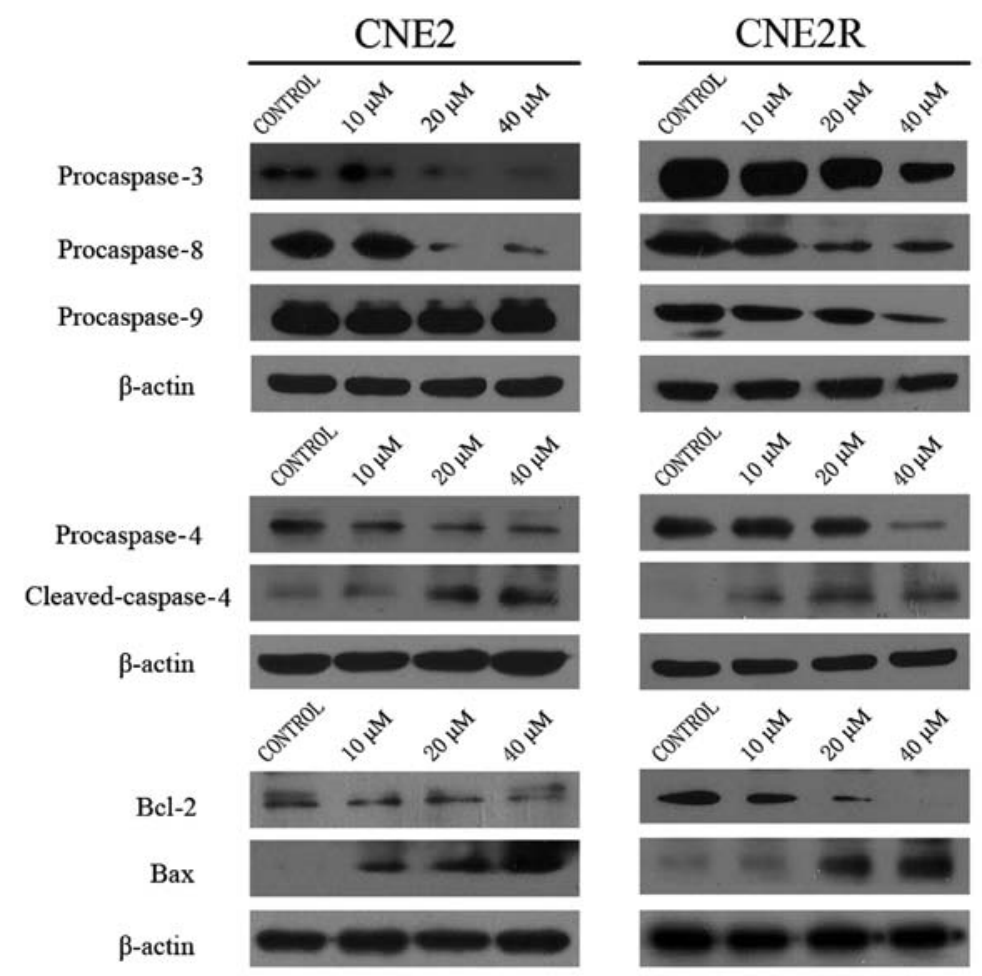

Figure 3. Activation of apoptosis in CNE2 and CNE2R cells by SZ-685C. Western blot analysis of procaspases-3, -8 , and -9 in CNE2 and CNE2R cells after SZ-685C treatment for $48 \mathrm{~h}$ with $12 \%$ polyacrylamide SDS gel. Bcl-2 and Bax were detected with $15 \%$ polyacrylamide SDS gel following SZ-685C treatment for 48 h. Procaspase- 4 and cleaved-caspase- 4 were detected following SZ-685C treatment for $24 \mathrm{~h}$. $\beta$-actin was used as an internal control.

A
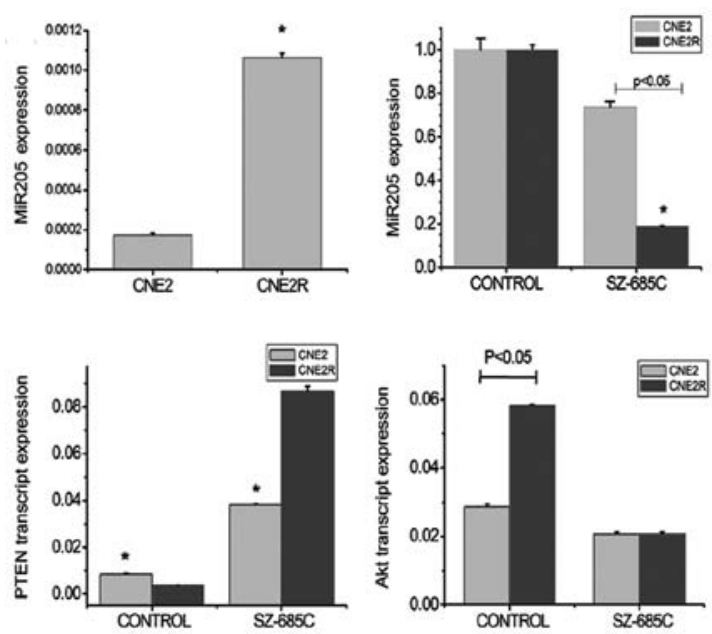

B

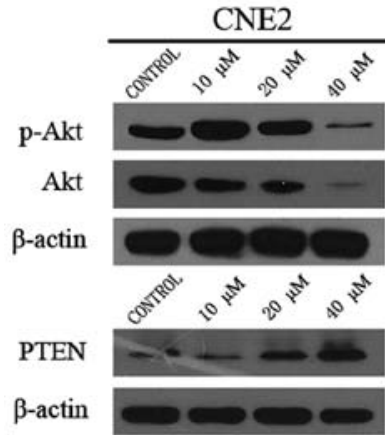

C
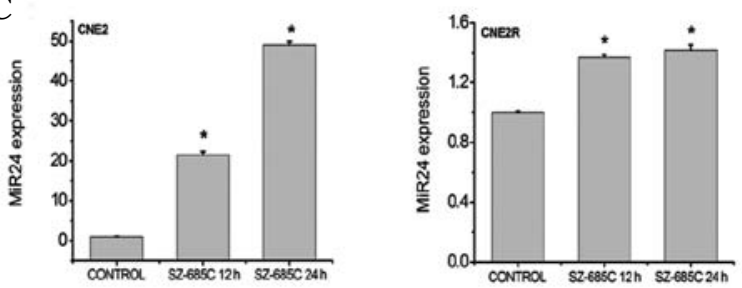

D

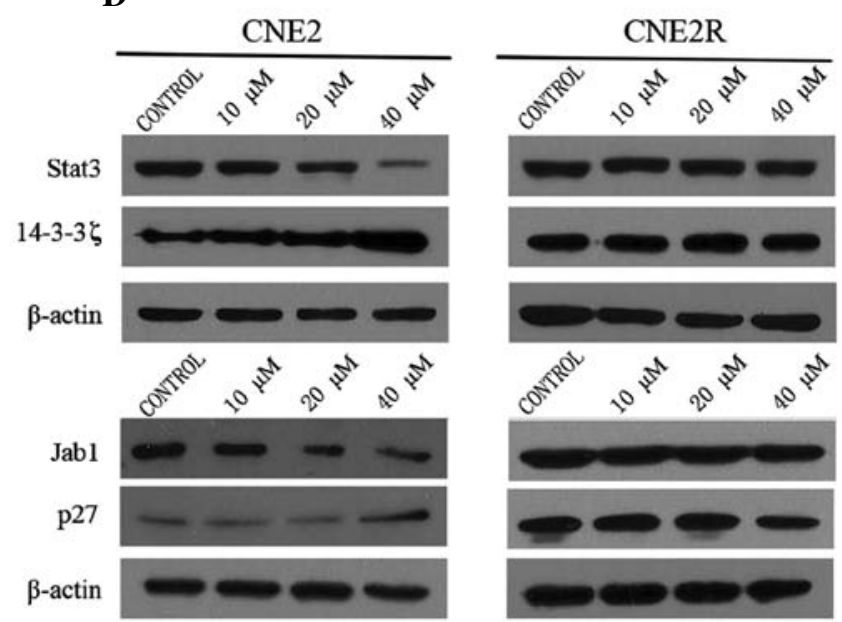

Figure 4. Effect of SZ-685C on the miR-205-PTEN-Akt and Stat3-Jab1-p27 pathways. (A) miR-205/PTEN. CNE2 and CNE2R cells were treated with SZ-685C for 4 and $1 \mathrm{~h}$ separately, and the expression levels were measured by qRT-PCR. Akt1 expression level was assayed at $48 \mathrm{~h}$ post-SZ-685C treatment in CNE2 and CNE2R cells. (B) p-Akt and Akt1 were detected by western blot analysis after SZ-685C treatment for $48 \mathrm{~h}$. The protein levels of PTEN in CNE2 and CNE2R cells treated with SZ-685C were measured at 4 and $1 \mathrm{~h}$. (C) miR-24 expression level was measured after treatment with SZ-685C for 12 and $24 \mathrm{~h}$ by qRT-PCR. (D) 14-3-3 $\zeta$ and Stat 3 were detected at $12 \mathrm{~h}$ post-SZ-685C treatment, and Jab1 and p27 were detected after SZ-685C treatment for $48 \mathrm{~h}$ by western blot analysis. 
(ER)-negative, invasive MD-MB-435 cells are highly sensitive to SZ-685C treatment. In addition, SZ-685C also represented a promising approach to the treatment of adriamycin-resistant breast cancer (13).

In the present study, we found that SZ-685C inhibited the viability of CNE2 and CNE2R cells with mild cytotoxicity. The $\mathrm{IC}_{50}$ value of CNE2 was higher than that of CNE2R for SZ-685C treatment for $24 \mathrm{~h}$. However, the results did not achieve a significant difference. The $\mathrm{IC}_{50}$ values in the two cell lines were almost the same at $72 \mathrm{~h}$. Our data indicated that SZ-685C exhibited a cytotoxic effect in both radioresistant and radiosensitive NPC cells. As we all know, the survival of cancer cells involves many factors, such as avoiding apoptosis and promoting cell cycle progression. We concluded that the anticancer activity of SZ-685C was mainly based on inducing apoptosis, partially resulting in cell cycle arrest (G2/M). The apoptotic pathways have been classified into two main types: extrinsic and intrinsic pathways. The extrinsic pathways include death receptor and survival factor withdrawal pathways, and the intrinsic pathways are caused either by DNA damage or stress to endoplasmic reticulum (ERS). Both pathways subsequently activate certain caspases. Activation of caspase- 8 and caspase- 4 seems to be involved in extrinsic pathways, while caspase- 4 is also linked with ERS-induced apoptosis. Activation of these specific capases ultimately leads to cell death through their effect on mitochondrial membrane stability and activation of caspase-9 $(14,15)$. In order to fully understand the pro-apoptotic mechanisms of SZ-685C, we analyzed the changes in various capsases. We found the activation of caspase- $3,-4$ and -8 in both cell lines. But the activation of caspase-9 was only found in CNE2R cells. We assumed that both extrinsic and intrinsic apoptotic pathways were activated after exposure to SZ-685C, but there was a slight difference between the two cell lines concerning the caspase-apoptotic pathways.

Numerous studies have demonstrated that Akt plays a pivotal role in chemotherapeutic resistance in many cancer types (16). Activation of Akt has been described in different tumors which are resistant to irradiation therapy (17), and PTEN is a negative factor for activation of Akt (18). Notably, Li et al reported that latent membrane protein 1 (LMP1) mediates the drug resistance of NPC cells to apoptosis by activation of the PI3K/Akt signaling pathway (19). Suppressing Akt activity may sensitize cancer cells to chemotherapy and irradiation (20). Importantly, research indicated that SZ-685C displayed marked pro-apoptotic activity by suppression of the Akt/FOXO pathway, which was related closely with cancer resistance. Therefore, these results provided reasonable evidence that SZ-685C induced apoptosis in NPC cells, particularly in radioresistant cells, through inhibition of the Akt pathway. Our data indicated that SZ-685C exhibited pro-apoptotic activity via inactivation of Akt and phosphorylation of Akt, and demonstrated that the inactivation of p-Akt was negatively regulated by PTEN. $\mathrm{Qu}$ et al (9) screened the differential expression of miRs between CNE2 and CNE2R cells and found that miR-205 was highly expressed in CNE2R cells. They also elucidated that miR-205 significantly upregulated Akt through targeting PTEN, resulting in the radioresistance of NPC cells. Based on our findings, we concluded that SZ-685C induced apoptosis by inactivating Akt through a decrease in miR-205 expression level which targeted PTEN, demonstrating that SZ-685C had an impact on the miR-205-PTEN-Akt pathway in both NPC cell lines. Yet, we found that the change in this pathway was more significant in CNE2R than that in CNE2 cells.

It was rational to declare that $\mathrm{SZ}-685 \mathrm{C}$ induced apoptosis through the miR-205-PTEN-Akt signaling pathway in CNE2R cells as the expression level of miR-205 was high. But we questioned why SZ-685C also showed a similar pro-apoptotic effect in CNE2 cells, in which the level of miR-205 was much lower than that of CNE2R cells, Was this the only pathway? To further elucidate the anticancer mechanisms in CNE2 cells, we assessed other probable anticancer pathway proteins. Notably, we found that various proteins related to apoptosis including 14-3-3\}, Stat3, Jab1 and p27 were altered in the CNE2 cells but not in the CNE2R cells. It has been confirmed that Jab1 plays a crucial role in the inactivation of several key tumor suppressors, such as p27, p53 and Smad 417 (21-23). Importantly, Jab1 and $\mathrm{p} 27$ protein levels were found to be inversely correlated in NPC cells (24). Upstream of Jab1, Stat3 acts as a positive regulator of Jabl. Its phosphorylation aids in its translocation to the nucleus, resulting in activation of Jab1 (25). Our findings suggest that SZ-685C downregulated the activation of Stat3 after SZ-685C treatment for $12 \mathrm{~h}$, and the level of p27 increased after treatment with SZ-685C for $48 \mathrm{~h}$. There is evidence that $14-3-3 \zeta$ as a chaperonin has a high affinity to Stat3. It is essential for activation of p-Stat3, binding to Ser727 position of Stat3 (26). However, we found that the level of 14-3-35 increased in CNE2 cells treated with SZ-685C for $12 \mathrm{~h}$. The helping role of $14-3-3 \zeta$ in the Stat3-Jab1-p27 pathway should be subsequently explored. A few studies have observed that the activation of Stat 3 and Akt concern parallel signaling pathways involved in tumor resistance, or carcinogenesis, migration and invasion. One study pointed out that the Akt signaling pathway is related to apoptosis, and the Stat 3 signaling pathway may be concerned with migration and invasion (27). There is also evidence to suggest that activation of Stat3 may facilitate the activation of Akt (28). After treatment with SZ-685C for $12 \mathrm{~h}$ in CNE2 cells, the level of Stat 3 was significantly decreased in a dose-dependent manner, and at the same time the level of total Akt maintained the same level (data not shown). We conjectured that activation of Stat 3 may occur before inactivation of Akt, but the mechanism between these two pathways should be explored next. Collectively, our data strongly suggest that SZ-685C regulates the Stat3-Jab1-p27 pathway, which is important to its anticancer effect in CNE2 cells.

We further studied changes in the expression of various miRs between the two cell lines after treatment with SZ-685C. Surprisingly, we found that expression of miR-24 was increased to nearly 50-fold upon SZ-685C treatment for $24 \mathrm{~h}$ in CNE2 cells. We proposed that miR-24 may be a key factor in the pro-apoptotic effect of SZ-685C on CNE2 cells. The relationship between miR-24 and Stat3-Jab1-p27 should be explored. We supposed that SZ-685C exhibited the pro-apoptotic activity in both radiosensitive and radioresistant NPC cells by regulating miRs leading to a series of changes in cell signaling pathways.

Taken together, we conclude that SZ-685C induced apoptosis in both radiosensitive and radioresistant NPC cell lines. We demonstrated that the pro-apoptotic mechanism of SZ-685C was associated with multi-signaling pathways, 
not only miR-205-PTEN-Akt but also Stat3-Jab1-p27, and that miR-24 may participate in the anticancer effect, but the exact mechanism still needs to be explored. Although various issues need to be further investigated, SZ-685C shows promise to become an effective candidate anticancer drug for NPC treatment.

\section{Acknowledgements}

This study was supported by the Guangdong Science and Technology Program (no. 2008A030201009). We thank Professor Z. She for providing the drug.

\section{References}

1. Her C: Nasopharyngeal cancer and the Southeast Asian patient. Am Fam Physician 63: 1776-1782, 2001.

2. Lo KW, To KF and Huang DP: Focus on nasopharyngeal carcinoma. Cancer Cell 5: 423-428, 2004.

3. Qin L, Zhang X, Zhang L, et al: Downregulation of BMI-1 enhances 5-fluorouracil-induced apoptosis in nasopharyngeal carcinoma cells. Biochem Biophys Res Commun 371: 531-535, 2008.

4. Schoof CR, Botelho EL, Izzotti A and Vasques Ldos R: MicroRNAs in cancer treatment and prognosis. Am J Cancer Res 2: 414-433, 2012.

5. Lee KM, Choi EJ and Kim IA: microRNA-7 increases radiosensitivity of human cancer cells with activated EGFR-associated signaling. Radiother Oncol 101: 171-176, 2011.

6. Jeong SH, Wu HG and Park WY: LIN28B confers radio-resistance through the posttranscriptional control of KRAS. Exp Mol Med 41: 912-918, 2009.

7. Tian W, Chen J, He H and Deng Y: MicroRNAs and drug resistance of breast cancer: basic evidence and clinical applications. Clin Transl Oncol: Aug 23, 2012 (Epub ahead of print).

8. Kanakkanthara A and Miller JH: MicroRNAs: novel mediators of resistance to microtubule-targeting agents. Cancer Treat Rev 39: 161-70, 2012.

9. Qu C, Liang Z, Huang J, et al: MiR-205 determines the radioresistance of human nasopharyngeal carcinoma by directly targeting PTEN. Cell Cycle 11: 785-796, 2012.

10. She Z, Chen S, Lin Y, Yuan J, Pang J and Li M: SZ-685C preparation method and antitumor application. Application No: 00810028628.3, Application Date: 2008.6.6.

11. Xie G, Zhu X, Li Q, et al: SZ-685C, a marine anthraquinone, is a potent inducer of apoptosis with anticancer activity by suppression of the Akt/FOXO pathway. Br J Pharmacol 159: 689-697, 2010 .
12. Zhang Y, Gan B, Liu D and Paik JH: FoxO family members in cancer. Cancer Biol Ther 12: 253-259, 2011.

13. Zhu X, He Z, Wu J, et al: A marine anthraquinone SZ-685C overrides adriamycin-resistance in breast cancer cells through suppressing Akt signaling. Mar Drugs 10: 694-711, 2012.

14. Hitomi J, Katayama T, Eguchi Y, et al: Involvement of caspase-4 in endoplasmic reticulum stress-induced apoptosis and Abetainduced cell death. J Cell Biol 165: 347-356, 2004.

15. Tadros SF, D'Souza M, Zhu X and Frisina RD: Apoptosis-related genes change their expression with age and hearing loss in the mouse cochlea. Apoptosis 13: 1303-1321, 2008.

16. LoPiccolo J, Granville CA, Gills JJ and Dennis PA: Targeting Akt in cancer therapy. Anticancer Drugs 18: 861-874, 2007.

17. Eke I, Koch U, Hehlgans S, et al: PINCH1 regulates Akt1 activation and enhances radio-resistance by inhibiting PP1alpha. J Clin Invest 120: 2516-2527, 2010.

18. Song MS, Salmena L and Pandolfi PP: The functions and regulation of the PTEN tumour suppressor. Nat Rev Mol Cell Biol 13: 283-296, 2012.

19. Li SS, Yang S, Wang S, Yang XM, Tang QL and Wang SH: Latent membrane protein 1 mediates the resistance of nasopharyngeal carcinoma cells to TRAIL-induced apoptosis by activation of the PI3K/Akt signaling pathway. Oncol Rep 26: 1573-1579, 2011.

20. Kraus AC, Ferber I, Bachmann SO, et al: In vitro chemo- and radio-resistance in small cell lung cancer correlates with cell adhesion and constitutive activation of AKT and MAP kinase pathways. Oncogene 21: 8683-8695, 2002.

21. Tomoda K, Kubota Y and Kato J: Degradation of the cyclindependent-kinase inhibitor p27Kip1 is instigated by Jab1. Nature 398: 160-165, 1999.

22. Oh W, Lee EW, Sung YH, et al: Jab1 induces the cytoplasmic localization and degradation of p53 in coordination with $\mathrm{Hdm} 2$. J Biol Chem 281: 17457-17465, 2006.

23. Wan M, Cao X, Wu Y, et al: Jab1 antagonizes TGF-beta signaling by inducing Smad4 degradation. EMBO Rep 3: 171-176, 2002.

24. Pan Y, Zhang Q, Tian L, et al: Jab1/CSN5 negatively regulates p27 and plays a role in the pathogenesis of nasopharyngeal carcinoma. Cancer Res 72: 1890-1900, 2012.

25. Shackleford TJ, Zhang Q, Tian L, et al: Stat3 and CCAAT/ enhancer binding protein beta (C/EBP-beta) regulate Jab1/CSN5 expression in mammary carcinoma cells. Breast Cancer Res 13: R65, 2011.

26. Zhang J, Chen F, Li W, et al: $14-3-3 \zeta$ interacts with stat 3 and regulates its constitutive activation in multiple myeloma cells. PLoS One 7: e29554, 2012.

27. Azijli K, Yuvaraj S, Peppelenbosch MP, et al: Kinome profiling of non-canonical TRAIL signaling reveals RIP1-Src-STAT3 dependent invasion in resistant non-small cell lung cancer cells. J Cell Sci 125: 4651-4661, 2012.

28. Chen F: JNK dependent Stat 3 phosphorylation contributes to Akt activation in response to arsenic exposure. Toxicol Sci 129: 363-371, 2012. 